\title{
A Case of Atypical Amyloid Polyneuropathy with Predominant Upper-limb Involvement with the Diagnosis Unexpectedly Found at Lung Operation
}

\author{
Yuichiro Shirota ${ }^{1}$, Atsushi Iwata ${ }^{1,4}$, Hiroyuki Ishiura ${ }^{1}$, Meiko Hashimoto ${ }^{1}$, Jun Goto ${ }^{1}$, \\ Jun Shimizu ${ }^{1}$, Ritsuko Hanajima ${ }^{1}$, Jun Nakajima ${ }^{2}$, Yutaka Takazawa ${ }^{3}$ and Shoji Tsuji ${ }^{1}$
}

\begin{abstract}
We present a patient of familial amyloid polyneuropathy (FAP) with predominant upper-limb involvement, the pattern of which resembled a mononeuropathy multiplex pattern. Sural nerve biopsy failed to diagnose the disorder, but lung partial resection performed later for other diagnostic purposes suggested FAP. A rare mutation in the transthyretin gene (S50R) was subsequently confirmed. Diagnostic challenges of FAP with atypical clinical presentations, including difficulties in pathological diagnosis, are discussed with a review of the literature.
\end{abstract}

Key words: familial amyloid polyneuropathy, transthyretin, mononeuropathy multiplex, nerve biopsy, lung partial resection

(Inter Med 49: 1627-1631, 2010)

(DOI: 10.2169/internalmedicine.49.3663)

\section{Introduction}

Familial amyloid polyneuropathy (FAP) patients usually develop small fiber neuropathy predominantly affecting the lower limbs or autonomic neuropathy between 20 and 40 years of age (1). Various atypical clinical presentations are reported (2-5), and the absence of amyloid deposits in the nerve biopsy specimen requires another measure to attain the correct diagnosis. In this report, we describe an upperlimb-predominant FAP patient with rare mutation of amyloidogenic transthyretin (ATTR), whose diagnosis was initially difficult, but was later suggested by the findings of lung partial resection conducted for other diagnostic purposes.

\section{Case Report}

A 57-year-old Japanese male from Shimane prefecture was referred to our hospital for evaluation of tingling, numbness, and poor dexterity of his hands. These symptoms developed in his right hand at the age of 51 and were progressive; similar symptoms also appeared in his left hand at the age of 56. Around the same time, mild dysesthesia occurred in his distal lower limbs, but this was neither disabling nor progressive. He lost ten kilograms in two years. Neurological examination on admission revealed weakness, muscle atrophies and decreased sensations at the right hand which mainly involved the median and ulnar nerve areas. Muscle strength was Medical Research Council (MRC) grade 2 in the right abductor pollicis brevis, 3 in the right interossei, and 4 in the right finger flexors and extensors. The lower extremities showed no weakness or atrophies, but distal dysesthesia was present. Tendon reflexes were notably diminished. He was free of autonomic dysfunctions, including orthostatic hypotension, syncope, gastrointestinal symptoms, urinary symptoms, sweating disorders, and sexual impotence. He used to drink about $250 \mathrm{~mL}$ of whisky and smoke 2 packs of cigarettes per day, but he had no disease history. His family history was negative for neuropathy or cardiac diseases.

\footnotetext{
${ }^{1}$ Department of Neurology, Graduate School of Medicine, the University of Tokyo, Tokyo, ${ }^{2}$ Department of Cardiothoracic Surgery, Graduate School of Medicine, the University of Tokyo, Tokyo, ${ }^{3}$ Department of Pathology, Graduate School of Medicine, the University of Tokyo, Tokyo and ${ }^{4}$ Department of Molecular Neuroscience on Neurodegeneration, the University of Tokyo, Tokyo

Received for publication March 15, 2010; Accepted for publication April 21, 2010

Correspondence to Dr. Shoji Tsuji, tsuji@m.u-tokyo.ac.jp
} 
Table 1. Nerve Conduction Study

\begin{tabular}{|c|c|c|c|c|}
\hline \multicolumn{5}{|l|}{ Sensory } \\
\hline Nerve & Stimulation site & Distal latency (ms) & Amplitude $(\mu \mathrm{V})$ & Velocity $(\mathrm{m} / \mathrm{s})$ \\
\hline $\mathbf{R}$ median & digit 2 & Absent & Absent & Absent \\
\hline L median & digit 2 & Absent & Absent & Absent \\
\hline R ulnar & digit 5 & Absent & Absent & Absent \\
\hline L ulnar & digit 5 & 2.7 & 0.4 & 40 \\
\hline R sural & ankle & 3.3 & 0.4 & 46 \\
\hline L sural & ankle & 3.1 & 1.3 & 48 \\
\hline \multicolumn{5}{|l|}{ Motor } \\
\hline Nerve & Stimulation site & Distal latency (ms) & Amplitude (mV) & Velocity $(\mathrm{m} / \mathrm{s})$ \\
\hline R median & wrist, elbow & Absent & Absent & Absent \\
\hline \multirow[t]{2}{*}{ L median } & wrist & 8.1 & 0.5 & \\
\hline & elbow & & 0.5 & 34 \\
\hline \multirow[t]{3}{*}{ R ulnar } & wrist & 4.5 & 4.1 & \\
\hline & below elbow & & 2.7 & 33 \\
\hline & above elbow & & 2.3 & 29 \\
\hline \multirow[t]{3}{*}{ L ulnar } & wrist & 3.0 & 9.6 & \\
\hline & below elbow & & 7.3 & 48 \\
\hline & above elbow & & 6.8 & 18 \\
\hline \multirow[t]{2}{*}{ R tibial } & ankle & 3.1 & 10.2 & \\
\hline & knee & & 6.7 & 42 \\
\hline \multirow[t]{2}{*}{ L tibial } & ankle & 3.2 & 10.3 & \\
\hline & knee & & 6.3 & 43 \\
\hline
\end{tabular}

Abnormal values are in Italic. L: left, R: right

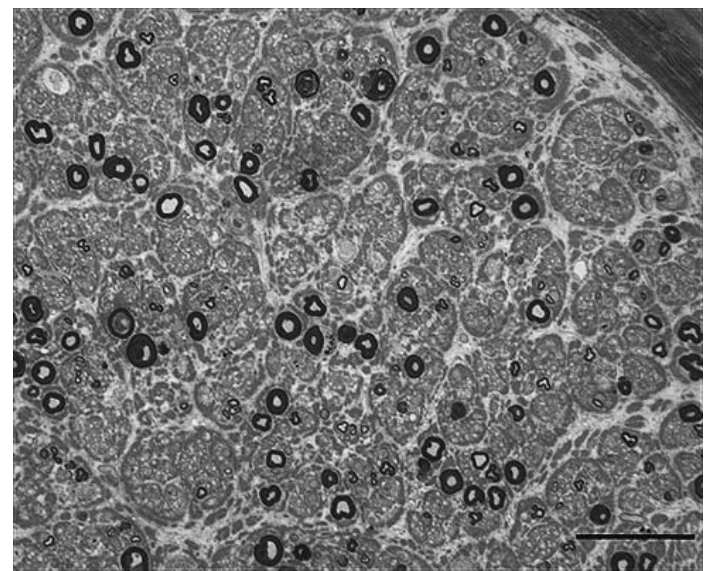

Figure 1. Sural nerve biopsy showed a moderate loss of myelinated fibers. A few myelin ovoids were recognized. Toluidine blue staining. Bar=50 $\mathrm{mm}$.

Nerve conduction study (NCS) revealed severe and diffuse damage in the sensory nerves, especially in the upper limbs (Table 1). With regard to the motor nerves, both of the median nerves were severely affected, and right ulnar nerve stimulation demonstrated an abnormal temporal dispersion and a decreased conduction velocity of $33 \mathrm{~m} / \mathrm{sec}$. Tibial nerves were normal, including maximal $\mathrm{F}$ wave conduction velocity of $57.0 \mathrm{~m} / \mathrm{s}$ (right) and $63.8 \mathrm{~m} / \mathrm{s}$ (left).
Right sural nerve biopsy was performed, which showed a reduction in myelinated fiber density and signs of acute axonal degeneration (Fig. 1). There were no signs of segmental demyelination, inflammation, or endoneurial edema. Congo red or methyl violet staining revealed no amyloid deposits. Computed tomography (CT) of the chest and abdomen was performed to determine whether some malignancy underlies his neuropathy or his weight loss, and revealed an 8-mm lung nodule which was thought as inflammatory change.

Although we could not reach a diagnosis, we considered the possibility of a variant of chronic inflammatory demyelinating polyneuropathy (CIDP), taking the mononeuropathy multiplex pattern into account; we performed intravenous immunoglobulin therapy without any effect. Afterwards, electron microscopy revealed obvious unmyelinated fiber loss, contrary to the diagnosis of CIDP. No amyloid deposition was evident. Thus, the etiology of the neuropathy remained unknown. The patient visited our clinic periodically with gradual worsening: owing to the deterioration of his leg symptoms, he developed the need for a cane, and he also lost his ability to use chopsticks. He had, however, no autonomic dysfunction clinically. Electrocardiogram revealed non-specific intraventricular conduction abnormality, and coefficent of variation in R-R intervals (CVR-R) was $2.83 \%$. Transthoracic echocardiogram showed left ventricular hy- 

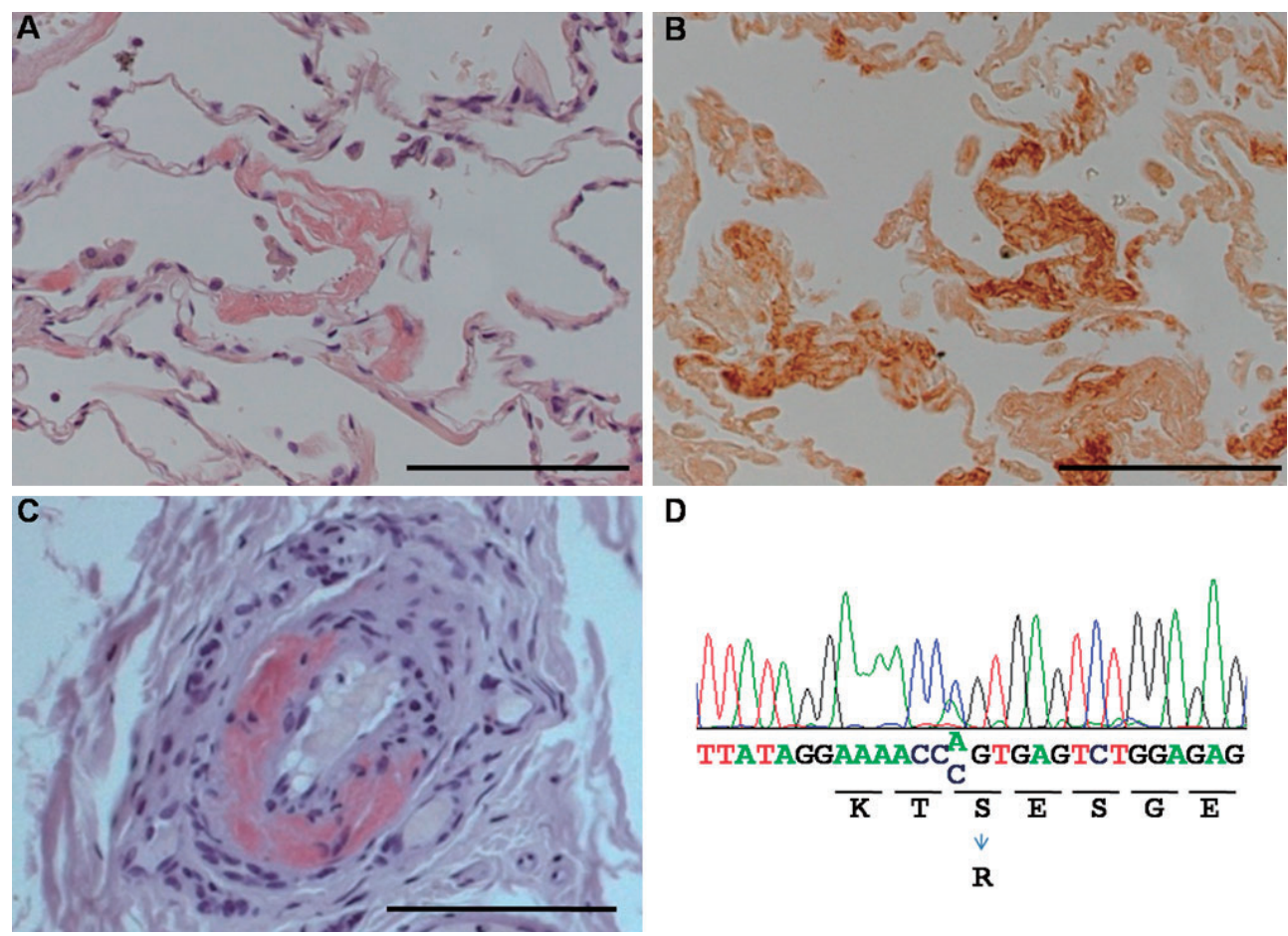

D

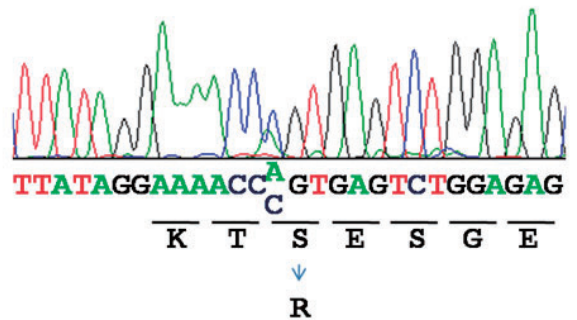

Figure 2. A: Lung histology demonstrated alveolar septal amyloid deposits. Congo red staining. Bar $=100 \mu \mathrm{m}$. B: Transthyretin immunostaining demonstrated that the amyloid was composed of transthyretin. Transthyretin immunostaining. Bar $=100 \mu \mathrm{m}$. C: Re-examined sural nerve specimen revealed a small amount of amyloid in the epineurial small vessels. Congo red staining. Bar=100 $\mu \mathrm{m}$. D: Transthyretin gene sequencing showed an A-to-C mutation in one allele in exon 3, which resulted in an S50R amino acid change. The upper line designates nucleotides and the lower line shows amino acids.

pertrophy.

Two years later, the lung nodule was found to be slightly enlarged to the size of $11 \mathrm{~mm}$ at a follow-up CT scan. Partial resection of the lung was performed through the thoracoscope, but we could not find any nodule in the resected specimen. Postoperative CT scan did not show any nodule, and the nature of the nodule remained unclear. Histopathological examination, however, revealed some amyloid deposits in the alveolar septal region as well as in the vascular region (Fig. 2A); the amyloid deposits were immunohistochemically positive for TTR (Fig. 2B). Molecular analysis of the TTR gene using the patient's leukocyte genomic DNA revealed an A-to-C mutation in exon 3 resulting in the substitution of Arg (CGT) for Ser (AGT) at codon 50 (S50 R, Fig. 2D). Given these results, we thoroughly reinvestigated the previously obtained peripheral nerve specimen, and a small amyloid deposit was identified in the vascular walls in the epineurium (Fig. 2C), which finally confirmed the diagnosis of FAP as the cause of his neuropathy. On the other hand, TTR immunohistochemistry (IHC) was negative probably because of the paucity of the amount of the amyloid deposit.

\section{Discussion}

In this report, we presented a patient presenting with an upper-limb predominant mononeuropathy multiplex with no autonomic dysfunctions or any family histories for FAP, who turned out to have FAP. Initial hand involvement in FAP patients is mainly due to bilateral carpal tunnel syndrome (CTS), and is considered to be a feature of non-V30 M ATTR types $(6,7)$. Although the present patient probably had bilateral CTS, the ulnar nerves showed smaller sensory nerve action potentials than those of the sural nerves. The findings suggested the presence of mononeuropathy multiplex, which is rare in FAP and has only been reported once (4), in contrast to frequent occurrence of polyneuropathy in FAP. In addition, upper-limb neuropathy other than CTS with or without lower-limb involvement in patients with ATTR V30M has been recently reported $(8,9)$, and upper-limb neuropathy may be an important clue to the diagnosis of FAP.

The demyelinating feature suggested by NCS has been reported as the most common pitfall in diagnosing sporadic cases of FAP (10), and in some late-onset FAP patients with ATTR V30M, the slowing of conduction velocity and prolongation of distal latency were reported to be conspicuous (11). In fact, the electrophysiological findings of the right ulnar nerve observed in our patient also hampered the correct diagnosis in our patient. The absence of right median compound muscle action potential, however, suggests that axonal degeneration is likely the main cause of the neuropathy in the present case. These electrophysiological findings have not been previously described in FAP patients with 
Table 2. Reported FAP Cases with ATTR S50R

\begin{tabular}{|c|c|c|c|c|c|c|c|c|}
\hline Patient No. & Onset $(\mathbf{y})$ & Sex & Country & FH & Initial symptom & Autonomic dysfunction & Cardiac manifestation & Ref \\
\hline 1 & 39 & $\mathbf{M}$ & Japan & N/A & General wasting & N/A & N/A & 17 \\
\hline 2 & 41 & $\mathbf{M}$ & Japan & - & Impotence & Impotence, $\mathbf{O H}, \mathbf{G I}$ & First-degree AV block, hypertrophy & 9 \\
\hline 3 & 50 & $\mathbf{F}$ & France & + & Lower limb paresthesia & GI & Normal ECG, Hypertrophy & 10 \\
\hline 4 & 38 & $\mathbf{F}$ & Vietnam & + & Dyspnea & GI & Restrictive cardiomyopathy & 11 \\
\hline 5 & 42 & $\mathbf{M}$ & Spain & + & Anorexia & OH, GI, impotence & N/A & 12 \\
\hline 6 & 48 & $\mathbf{M}$ & Spain & + & Polyneuropathy & N/A & N/A & 12 \\
\hline 7 & 45 & $\mathbf{F}$ & Spain & + & Anorexia & GI & N/A & 12 \\
\hline 8 & 53 & $\mathbf{M}$ & Spain & + & Vitrous deposits in eyes & None & N/A & 12 \\
\hline 9 & 40 & $\mathbf{M}$ & Spain & + & Polyneuropathy & N/A & N/A & 12 \\
\hline 10 & 48 & $\mathbf{M}$ & Portugal & + & Pain in legs and feet & Impotence, GI, urinary incontinence & Third-degree AV block, normal TTE & 12 \\
\hline 11 & 68 & $\mathbf{F}$ & Portugal & + & Loss of sensation in feet & GI, $\mathrm{OH}$, urinary incontinence & N/A & 12 \\
\hline This case & 51 & $\mathbf{M}$ & Japan & - & Right hand dysesthesia & None & Hypertrophy & - \\
\hline
\end{tabular}

AV block: atrioventricular block, FH: family history, GI: gastrointestinal tract dysfunction (including constipation and diarrhea), N/A: information not available, OH: orthostatic hypotension, Ref: reference, TTE: transthoracic echocardiogram

Patients 5-9 are from the same family. Patients 10 and 11 are from the same family.

\section{ATTR S50R.}

Another atypical clinical feature of the present case was the absence of autonomic dysfunctions. Misu et al reported that five of the 35 late onset Japanese FAP cases with ATTR V30M did not show autonomic symptoms (12). Another study also confirmed that late-onset patients did not note autonomic symptoms in the initial phase, but showed them in the later phase (13). However, patients with ATTR S50R have been reported to frequently present with substantial autonomic symptoms (14-17). The present patient is the first FAP case of ATTR S50R with no obvious autonomic dysfunction in his disease course lasting as long as 8 years, although absence of autonomic dysfunction is sometimes difficult to demonstrate, and a more vigorous investigation such as the Shellong test might have revealed some abnormality.

Diagnostic difficulties on nerve biopsies deserve further discussion. Previous autopsy studies reported that the proximal portion of the peripheral nerve showed more amyloid deposition than the distal portion. Therefore, considering the small amount of amyloid deposit found in the specimen (i.e. distal portion of the nerve), the cause of the neuropathy in the present patient might be ascribed to possible deposition of amyloid in the more proximal region of the peripheral nerve (18). With regard to biopsy of the distal portion of the nerve, a retrospective study demonstrated that six of the 35 peripheral nerve biopsies obtained from patients with amyloid neuropathy required electron microscopy to detect an amyloid deposit, although 4 of the six reinvestigations of the specimen using light microscope could reveal it (19). Another study demonstrated that TTR IHC could reveal amyloid deposits in the sural nerve that could not be detected by Congo red staining, supporting increased sensitivity of TTR IHC (20). In the present case neither electron microscopic observation nor TTR IHC detected amyloid deposition. The distribution of amyloid deposits was also characteristic in our case. Amyloid deposits are usually found in the endoneurium, but in our case they were restricted to the vascular walls of the epineurium. Although several studies have shown epineurial amyloid deposition $(19,21)$, its frequency is as low as two out of 40 (21). The two cases, in which epineurial amyloid deposition was noted, were elderly (68 and 73 years old) and showed amyloid deposits only around the capillaries as in our patient.

The diagnosis of FAP patients often requires a tissue specimen other than peripheral nerves such as rectal or fat tissue; however, lung tissue is almost always the last to be obtained for FAP diagnosis. Note that there was as much amyloid deposition in the alveolar septal region as in the vascular walls. An autopsy series demonstrated that alveolar amyloid deposition is more often observed in elderly patients with FAP (22). Thus, the uncommon deposition pattern, that is, in the alveolar septum in the lung and in the epineurium in the peripheral nerve, may be due to aging or a rare mutation of S50R. In fact, another non-V30M mutation (D38A) was reported to demonstrate diffuse pulmonary amyloid deposition (23), indicating a possible correlation between non-V30M ATTR and pathological findings of diffuse pulmonary amyloid deposition.

How could we diagnose FAP earlier in the present patient? At least two issues should have been addressed. First, if we had considered neuropathy and a cardiac (i.e. ECG or echocardiographic) abnormality derived from the same origin, the possibility of FAP might have been explored further. A previous study revealed that patients with non-V30M ATTR often present with cardiac amylodosis (24). Second, to further explore the etiology of the neuropathy, it would be useful to include rectal or fat biopsies, which are less invasive than lung partial resection.

ATTR S50R is a rare mutation and has been mentioned in only five other reports in the literature $(14-17,25)$. In general, the patients with an ATTR S50R mutation in the literature showed more typical presentations as FAP (Table 2), hence our patient added another spectrum of FAP with ATTR S50R mutation as discussed above. Further investiga- 
tion of the phenotype-genotype correlation in additional cases will be needed.

In conclusion, we emphasize the diverse clinical presentations and the diagnostic challenge of FAP associated with an ATTR S50R mutation. Because FAP can be treated with liver transplantation earlier in the course, we should conduct vigorous investigations when we encounter apparently sporadic, immunotherapy-resistant patients with atypical clinical presentations such as mononeuropathy multiplex predominantly involving the upper limbs.

\section{Acknowledgement}

This work was partially supported in part by: KAKENHI (Grant-in-Aid for Scientific Research) on Priority Areas, Applied Genomics, and Global COE Program (Comprehensive center of education and research for chemical biology of the diseases) from the Ministry of Education, Culture, Sports, Science and Technology; Mochida Memorial Foundation for Medical and Pharmaceutical Research; and Sankyo Foundation of Life Science.

This work originated from the University of Tokyo.

This work was partially supported in part by: KAKENHI (Grant-in-Aid for Scientific Research) on Priority Areas, Applied Genomics, and Global COE Program (Comprehensive center of education and research for chemical biology of the diseases) from the Ministry of Education, Culture, Sports, Science and Technology; Mochida Memorial Foundation for Medical and Pharmaceutical Research; and Sankyo Foundation of Life Science.

\section{References}

1. Benson MD, Kincaid JC. The molecular biology and clinical features of amyloid neuropathy. Muscle Nerve 36: 411-423, 2007.

2. Nagasaka T, Togashi S, Watanabe H, et al. Clinical and histopathological features of progressive-type familial amyloidotic polyneuropathy with TTR Lys54. J Neurol Sci 276: 88-94, 2009.

3. Yazaki M, Yamashita T, Kincaid JC, et al. Rapidly progressive amyloid polyneuropathy associated with a novel variant transthyretin serine 25. Muscle Nerve 25: 244-250, 2002.

4. Briemberg HR, Amato AA. Transthyretin amyloidosis presenting with multifocal demyelinating mononeuropathies. Muscle Nerve 29: 318-322, 2004.

5. Salvi F, Scaglione C, Michelucci R, et al. Atypical familial motor neuropathy in patients with mutant TTR Ile68Leu. Amyloid 10: 185-189, 2003.

6. Mahloudji M, Teasdall RD, Adamkiewicz JJ, Hartmann WH, Lambird PA, McKusick VA. The genetic amyloidoses, with particular reference to hereditary neuropathic amyloidosis, Type II (Indiana or Rukavina type). Medicine 48: 1-37, 1969.

7. Ikeda S, Nakazato M, Ando Y, Sobue G. Familial transthyretintype amyloid polyneuropathy in Japan: Clinical and genetic heterogeneity. Neurology 58: 1001-1007, 2002.

8. Koike H, Morozumi S, Kawagashira Y, et al. The significance of carpal tunnel syndrome in transthyretin Val30Met familial amyloid polyneuropathy. Amyloid 16: 142-148, 2009.

9. Tojo K, Tsuchiya-Suzuki A, Sekijima Y, Morita H, Sumita N, Ikeda S. Upper limb neuropathy such as carpal tunnel syndrome as an initial manifestation of ATTR Val30Met familial amyloid polyneuropathy. Amyloid 17: 32-35, 2010.

10. Planté-Bordeneuve V, Ferreira A, Lalu T, et al. Diagnostic pitfalls in sporadic transthyretin familial amyloid polyneuropathy (TTRFAP). Neurology 69: 693-698, 2007.

11. Koike H, Kawagashira Y, Iijima M, et al. Electrophysiological features of late-onset transthyretin Met 30 familial amyloid polyneuropathy unrelated to endemic foci. J Neurol 255: 1526-1533, 2008.

12. Misu K, Hattori N, Nagamatsu M, et al. Late-onset familial amyloid polyneuropathy type I (transthyretin Met30-associated familial amyloid polyneuropathy) unrelated to endemic focus in Japan: Clinicopathological and genetic features. Brain 122: 1951-1962, 1999.

13. Koike H, Misu K, Ikeda S, et al. Type I (transthyretin Met 30) familial amyloid polyneuropathy in Japan. Early- vs late-onset form
Arch Neurol 59: 1771-1776, 2002.

14. Takahashi N, Ueno S, Uemichi T, Fujimura H, Yorifuji S, Tarui S. Amyloid polyneuropathy with transthyretin Arg50 in a Japanese case from Osaka. J Neurol Sci 112: 58-64, 1992.

15. Reilly MM, Adams D, Booth DR, et al. Transthyretin gene analysis in European patients with suspected familial amyloid polyneuropathy. Brain 118: 849-856, 1995.

16. Singer R, Schnabel A, Strasser RH. Restrictive cardiomyopathy in familial amyloidosis TTR-Arg-50. Circulation 107: 643-644, 2003.

17. Munar-Qués M, Masjuan J, Coelho T, Moreira P, Viader-Farré C, Saraiva MJM. Familial amyloid polyneuropathy associated with TTR Ser50Arg mutation in two Iberian families presenting a novel single base change in the mutant gene. Amyloid 14: 147-152, 2007.

18. Hanyu N, Ikeda S, Nakadai A, Yanagisawa N, Powell HC. Peripheral nerve pathological findings in familial amyloid polyneuropathy: a correlative study of proximal sciatic nerve and sural nerve lesions. Ann Neurol 25: 340-350, 1989.

19. Vital C, Vital A, Bouillot-Eimer S, Brechenmacher C, Ferrer X, Lagueny A. Amyloid neuropathy: a retrospective study of 35 peripheral nerve biopsies. J Peripher Nerv Syst 9: 232-241, 2004.

20. Sousa MM, Cardoso I, Femandes R, Guimarães A, Saraiva MJ. Deposition of transthyretin in early stages of familial amyloidotic polyneuropathy. Evidence for toxicity of nonfibrillar aggregates. Am J Pathol 159: 1993-2000, 2001.

21. Said G, Planté-Bordeneuve V. Familial amyloid polyneuropathy. A clinico-pathologic study. J Neurol Sci 284: 149-154, 2009.

22. Ueda $M$, Ando $Y$, Haraoka $K$, et al. Aging and transthyretinrelated amyloidosis: Pathologic examinations in pulmonary amyloidosis. Amyloid 13: 24-30, 2006.

23. Yazaki M, Takei Y, Katoh M, Ikeda S. Postmortem findings in two familial amyloidosis patients with transthyretin variant Asp38 Ala. Amyloid 7: 270-277, 2000.

24. Hattori T, Takei Y, Koyama J, Nakazato M, Ikeda S. Clinical and pathological studies of cardiac amyloidosis in transthyretin type familial amyloid polyneuropathy. Amyloid 10: 229-239, 2003.

25. Ueno S, Uemichi T, Takahashi N, Soga F, Yorifuji S, Tarui S. Two novel variants of transthyretin identified in Japanese cases with familial amyloidotic polyneuropathy: transthyretin (Glu42 to Gly) and transthyretin (Ser50 to Arg). Biochem Biophys Res Commun 169: 1117-1121, 1990.

(C) 2010 The Japanese Society of Internal Medicine http://www.naika.or.jp/imindex.html 